\title{
Level of selected pro-inflammatory markers in middle-aged women with Hashimoto's disease
}

\author{
Joanna Szczepanik', Sławomir Graczyk², Katarzyna Domaszewska ${ }^{2,3}$ \\ ${ }^{1}$ Nutrition Clinic Vitamin, Poznan, Poland \\ ${ }^{2}$ Faculty of Health Sciences, The President Stanislaw Wojciechowski State University of Applied Sciences in Kalisz, Poland \\ ${ }^{3}$ Department of Physiology and Biochemistry, Poznan University of Physical Education, Poland
}

\begin{abstract}
Introduction: Hashimoto's disease is an autoimmune disease in which the body produces antibodies against its own tissues, in this case against thyrocytes. Autoimmune diseases are associated with impaired immune tolerance. The aim of this study was to analyse selected pro-inflammatory markers in women with Hashimoto's (HT) autoimmune thyroiditis compared to a group of healthy women.

Material and methods: In this research study 41 women with HT were compared with 30 healthy women. The content of immunological markers was assessed in blood serum by enzyme-linked ELISA methods. Cytokine IL-1ß, IL-6, and TNF- $\alpha$ were assessed.

Results: The concentration of IL- $1 \beta$ for women with HT was 3.1 (1.8-4.9) pg/ml, and for healthy women $1.0(0.1-1.9) \mathrm{pg} / \mathrm{ml}$. The concentration of IL-6 for women with HT was 12.1 (6.7-19.4) $\mathrm{pg} / \mathrm{ml}$, and for healthy women 3.8 (2.5-6.7) $\mathrm{pg} / \mathrm{ml}$. TNF- $\alpha$ concentration was higher for people with HT, at 3.4 (2.04.4) $\mathrm{pg} / \mathrm{ml}$, compared to healthy women, who had a level of $2.2(1.2-3.4) \mathrm{pg} / \mathrm{ml})$, but without significant statistical differences.

Conclusions: In HT we observe an increased immune response associated with an elevated concentration of pro-inflammatory cytokines.
\end{abstract}

KEY WORDS: interleukin 1 (IL-1 $\beta$ ), interleukin 6 (IL-6), human tumour necrosis factor alpha (TNF- $\alpha$ ), Hashimoto disease.

ADDRESS FOR CORRESPONDENCE: Katarzyna Domaszewska, Department of Physiology and Biochemistry, Poznan University of Physical Education, 27/39 Królowej Jadwigi St., 61-871 Poznan, Poland, e-mail: domaszewska@awf.poznan.pl

\section{INTRODUCTION}

Cytokines are glycoproteins that are released through the activation of cells of various tissues. They play a role in regulating many processes, such as differentiation, proliferation, or secretion of important biological substances. They modulate the immune response, balancing the body's own tolerance and blocking self-aggression. In addition, cytokines participate in repair processes, tissue healing, reduce inflammation, have hormone-like properties, and are involved in the haematopoietic process [1]. In 1956 Fromm et al. [2] pointed out that people with Hashimoto's disease appear to have elevated serum gamma globulin titres, i.e. disorders in the modulation of immune processes. Roitt et al. [3] found that this disease is associated with elevated thyroglobulin antibodies.
Immune response disorders, especially the high titre of anti-thyroid oxidase (aTPO) and thyroglobulin (aTG) antibodies, play a significant role in the pathogenesis of the disease. Antibodies whose titre is elevated simultaneously become cytotoxic agents and activators of cytotoxic $\mathrm{T}$ lymphocytes, leading to the destruction (apoptosis) of thyroid follicular cells [1]. In addition to the mentioned activation, the number of plasma cells increases, and the result of abnormal lymphocyte activity is the increased excretion of inflammatory cytokines: interleukin (IL)-1, IL-2, IL-6, tumour necrosis factor (TNF)- $\alpha$, and interferon (IFN)- $\gamma$, while reducing the cytokines that shape immune tolerance: IL-4, IL-5, and IL-10. Increased apoptosis of thyroid epithelial cells is the result of increased proinflammatory cytokine activity [1]. 
Cytokine production is carried out by lymphocytes, but also by thyroid follicular cells. In vitro studies confirm that as a result of IL- $1 \beta$, IFN- $\gamma$, and TNF- $\alpha$ stimulation, cytokine production by thyroid follicular cells occurs. This suggests an increase in pro-inflammatory activity and an increase in lymphatic infiltration in vivo. IL- $1 \beta$, IFN- $\gamma$, and TNF- $\alpha$ have the ability to increase the expression of adhesion molecules on the surface of thyroid follicular cells, while also stimulating the production of nitric oxide (NO) and prostaglandins (PG), enhancing the inflammatory response in autoimmune thyroid diseases. Cytokines, by presenting antigens to $\mathrm{T}$ lymphocytes in thyroid tissue, lead to the destruction of this gland (T-cell cytotoxicity). Based on thyroid tissue tests, an increased amount of IL-1, IL- 6 , and TNF- $\alpha$ was found in thyroid follicular cells. And in cell cultures, IL-1 and IL-6 are responsible for the proliferation of thyroid follicular cells, as well as inhibiting thyrocytes, stimulated by TSH [4]. Depending on the type and target of action, cytokines have a very broad activity, they can perform both immunostimulatory and immunosuppressive functions.

Cytokines such as IL-1 $\beta$, IL- 6 , and TNF- $\alpha$ stimulate inflammation and autoimmunity [5]. Their role is to increase the inflammatory response due to stimulation of $\mathrm{T}$ and $\mathrm{B}$ lymphocytes, causing the production of antibodies and damage to thyroid tissue through cell death, especially in Hashimoto's disease [6].

The aim of this study was to assess if there is a difference in the concentration of selected proinflammatory cytokines between people with Hashimoto's disease and healthy people.

\section{MATERIAL AND METHODS}

The study involved 41 women with Hashimoto's disease (based on a medical diagnosis) and 30 healthy women as a control group. The control group was selected for the test in terms of sex, weight, and height. Patients were excluded if they had at least one of the following: no obesity, being on a vegetarian or any other alternative diet, active or post-cancerous disease (ongoing radiation/chemotherapy treatment), liver diseases (ALT $>3 \times$ border line) except for patients with fatty liver disease, chronic kidney disease eGFR $<30 \mathrm{ml} / 1.73 \mathrm{~m}^{2} / \mathrm{min}$, acute inflammation CRP > $5 \mathrm{mg} / \mathrm{dl}$, unstable ischaemic heart disease, having an ischemic or haemorrhagic stroke in the past ( $<6$ months), having STEMI with a drug-eluting stent implantation, nSTEMI ( $<12$ months), inherited metabolic disorders: phenylketonuria, galactosaemia, autoimmune diseases (an acute thyroiditis, celiac disease, systemic connective tissue disease, haemolytic anaemia, vitiligo, Addison's disease, hyperbilirubinaemia), non-specific enteritis (Crohn's disease, ulcerative colitis), pregnancy, psychological disorders, eating disorders such as anorexia and bulimia, antibiotic therapy, steroid therapy (ongoing), and drug or alcohol addic- tion (a daily consumption of more than one portion of alcohol). Participants were qualified to the study group based on a medical diagnosis, which assumed the presence of Hashimoto's disease based on elevated thyroid antibody titres. The team did not re-examine the antibody titre because their elevated titre at one time point implies disease. Patients' declarations showed that their thyroid hormone test results were normal (fT4, fT3) as well as their hormone regulating thyroid function (TSH) by taking levothyroxine substitution. The control group had excluded the occurrence of thyroid diseases on the basis of laboratory tests. Their level of hormones responsible for the proper functioning of the thyroid gland were normal. Women belonging to the control group did not suffer from any autoimmune disease or chronic inflammation.

The respondents agreed to participate in the study and were informed about the voluntary nature, aims, benefits, and course of the study. The study was conducted according to the Declaration of Helsinki and the National Statement and Human Research Ethics Guidelines, and was approved by the IRB (Institute for Research in Biomedicine) at the Poznan University of Medical Sciences (2013-05-10; Ethics Approval Number: 302/13).

\section{PREPARATION OF BLOOD SAMPLES FOR ANALYSIS}

The participants were asked to stop supplementation that could potentially affect the result. They were asked not to do physical activity during the day before the examination. Blood samples were taken from the ulnar vein using an S-Monovette syringe (SARSTEDT, Nümbrecht, Germany) then placed in tubes containing a clot activator and centrifuged $\left(1500 \mathrm{~g}, 4^{\circ} \mathrm{C}, 4\right.$ minutes) to separate the serum. (Universal 320R; Hettich Lab Technology, Tuttlingen, Germany). Samples were frozen and stored at $-80^{\circ} \mathrm{C}$ until analysis (U410, Ultra Low Temperature Freezer, New Brunswick Scientific, USA).

\section{DETERMINATION OF IL-1SS, IL-6, AND TNF- $\alpha$ IN BLOOD SERUM}

The concentrations of IL- $1 \beta$, IL- 6 , and TNF- $\alpha$ were measured with the use of the ELISA immunoenzymatic method (DRG International, Inc., USA). The sensitivity of the ELISA kits was as follows: $0.35 \mathrm{pg} / \mathrm{ml}, 0.2 \mathrm{pg} / \mathrm{ml}$, and $0.7 \mathrm{pg} / \mathrm{ml}$. The intra- and inter-assay coefficients of variation $(\mathrm{CVs})$ were less than $10 \%$. The samples were read using a Synergy 2 SIAFRT multi-detection microplate reader from BioTek (USA) at the manufacturer's recommended wavelength.

\section{STATISTICAL ANALYSIS}

All data are presented as means and standard deviations (SD). The normality of the distribution was tested by the Shapiro-Wilk test. Differences between vari- 
ables (for variables with non-normal distribution) were examined using the Mann-Whitney U test. Spearman rank analysis was used to calculate the correlation coefficients. The level of statistical significance was set at $p<0.05$. Dell Inc. software was used for statistical analysis (2016) Dell Statistica 13 (Tulsa, Oklahoma, USA).

\section{RESULTS}

The results of anthropometric measurements of participants can be found in Table 1 . The examined groups did not differ significantly in terms of weight, body height, and BMI. Women with Hashimoto's thyroiditis (HT) were characterised by a significantly higher concentration of IL- $1 \beta$ and IL- 6 in the blood and an insignificantly higher concentration of TNF- $\alpha$ (Table 1), compared to the control group. Correlation analysis of blood biochemical results obtained from both groups showed a significant relationship between IL- $1 \beta$ and IL- 6 $(r=0.4696, p<0.0001)$, IL-1 $\beta$ and TNF- $\alpha(r=0.2969$, $p<0.05)$, and IL-6 and TNF- $\alpha(r=0.3126, p<0.01)$. A significant relationship between IL- 6 and TNF- $\alpha$ at the level $r=0.4844, p<0.01$ was demonstrated only in the group of healthy women, and in the group of women with Hashimoto's disease a significant relationship between age and IL- 6 blood concentration $(r=-0.3191$, $p<0.05)$ was shown.

\section{DISCUSSION}

HT is a very common organ-specific autoimmune disease, characterised by the presence of circulating thyroid antibodies and infiltration by autoreactive lymphocytes of the thyroid gland. It is still not completely clear how HT is formed. It seems to be the result of interaction between environmental factors and genetic susceptibility, which both lead to the breakdown of natural self-antigen tolerance and to an increased activity of autoreactive lymphocytes and immunoglobulins. This study focuses on comparing the concentration of selected proinflammatory cytokines (IL- $1 \beta$, IL- 6 , and TNF- $\alpha$ ) in people with HT compared to healthy people. The introduction mentions that cytokines such as IL-1 $\beta$, IL- 6 , and TNF- $\alpha$ stimulate inflammation and autoimmunity, due to stimulation of $\mathrm{T}$ and $\mathrm{B}$ lymphocytes, causing the production of antibodies and damage to thyroid tissue through cell death [6] .

In autoimmune thyroid diseases both Th1 and Th2 cells play an important role in the immune response of the system. The Th1 cells produce pro-inflammatory cytokines: IL-1 $\beta$, IL-2, TNF- $\alpha$, and IFN- $\gamma$. However, the Th2 response with IL-4, IL-5, IL-6, IL-9, IL-10, and IL-13 production also occurs. Cytokines produced by both Th1 and Th 2 cells have an inhibitory effect on each other. Th1 is involved in the inflammation and cellular immune response, while Th2 is a stimulator of antibody production, participates in the humoral immune response, and also promotes allergic and immune responses [1].

In this study, it was shown that women with HT, compared to the control group, had significantly higher levels of IL-1 $\beta$ and IL- 6 in the blood, and insignificantly higher levels of TNF- $\alpha$. IL- 6 can have pro-inflammatory and anti-inflammatory effects. Its anti-inflammatory effect is due to its ability to inhibit the inflammatory response by reducing the synthesis of TNF- $\alpha$ and IL- $1 \beta$ and affecting chemokine expression. On the other hand, TNF- $\alpha$ and IL- $1 \beta$ are markers that cause a significant increase in the expression of IL-6, as well as other cytokines from its family [7]. In acute inflammation, the role of IL-6 is to reduce the inflammatory response; however, in chronic inflammation, it mainly has pro-inflammatory properties. This may explain its higher rate in our study because HT is associated with chronic thyroid inflammation. Other authors also confirm that in autoimmune thyroid diseases there is an increased concentration of IL-6, which is produced by thyroid follicular cells [8-10]. Studies of the Indian, Chinese, and Japanese populations have shown that some polymorphisms within cytokine genes such as IFN- $\gamma$, IL- 6 , or TNF have a positive correlation with the development of HT [11-13]. We did not assess polymorphisms, but the higher concentrations of selected cytokines may indicate that the genetic factors responsible for their production are of great importance

TABLE 1. Anthropometric characteristics of the studied women and the average concentration of pro-inflammatory blood indicators tested

\begin{tabular}{|l|c|c|c|}
\hline Characteristics & $\begin{array}{c}\text { Hashimoto's thyroiditis women } \\
(n=41)\end{array}$ & $\begin{array}{c}\text { Control group } \\
(n=30)\end{array}$ & $p$-value \\
\hline Age (years) & $40.0(32.0-49.0)$ & $41.0(34.0-55.0)$ & NS \\
\hline Body weight $(\mathrm{kg})$ & $61.0(55.0-70.0)$ & $64.5(58.0-72.0)$ & NS \\
\hline Body height $(\mathrm{cm})$ & $169.0(164.0-172.0)$ & $168.0(163.0-170.0)$ & NS \\
\hline BMI $\left(\mathrm{kg} / \mathrm{m}^{2}\right)$ & $23.2(19.3-24.7)$ & $22.1(20.4-26.3)$ & NS \\
\hline IL-1 $\beta(\mathrm{pg} / \mathrm{ml})$ & $3.1(1.8-4.9)$ & $1.0(0.1-1.9)$ & $<0.001$ \\
\hline IL-6 $(\mathrm{pg} / \mathrm{ml})$ & $12.1(6.7-19.4)$ & $3.8(2.5-6.7)$ & $<0.001$ \\
\hline TNF- $\alpha(\mathrm{pg} / \mathrm{ml})$ & $3.4(2.0-4.4)$ & $2.2(1.2-3.4)$ & NS \\
\hline
\end{tabular}

Results are expressed as median (interquartile range). The significance tests used are the Mann-Whitney $U$ test for non-normally distributed variables. NS - not statistically significant 
in Hashimoto's disease. This has also been suggested by other Polish researchers [14].

IL- $1 \beta$ is released by peripheral blood monocytes, stimulates $\mathrm{T}$ lymphocytes to synthesize other proinflammatory cytokines, and affects the proliferation and differentiation of B lymphocytes. In addition, it stimulates the release of growth and chemotactic factors, inducing the migration of phage cells and promoting local inflammation. By increasing body temperature, it works systemically and increases the production of acute-phase proteins [15].

TNF- $\alpha$ is an acute phase protein that initiates a cytokine cascade, increasing vascular permeability, and it plays a role in inflammatory and immune responses. As a result of stimulation by other cytokines, e.g. IL- $1 \beta$, TNF- $\alpha$ is also directly released [16]. Lei et al. assessed the level of selected proinflammatory factors in people with thyroid autoimmune diseases, euthyreosis, and hypofunction, compared to healthy people. They proved that TNF- $\alpha$ levels were higher for people with hypothyroidism compared to people with levelled thyroid hormones. Interestingly, this concentration showed a decreasing relationship for hypothyroidism and euthyreosis in the group of sick people and among healthy people [17]. Results from as early as the 1990s suggested that TNF- $\alpha$ is present in the thyroid tissue of its various diseases. Also, thyroid-derived lymphocytes are potential producers of TNF- $\alpha$, which can locally affect thyroid function [18].

Cytokines such as IL-1 $\beta$, TNF- $\alpha$, and IL- 6 play a key role in modulating the immune response in an autoimmune disorder and are therefore considered as markers of hypothyroidism [19].

\section{CONCLUSIONS}

A statistically significantly higher concentration of proinflammatory cytokines IL-1 $\beta$ and IL- 6 , and a non-statistically significantly higher concentration of TNF- $\alpha$ was found among participants with HT compared to healthy controls.

\section{DISCLOSURE}

The authors report no conflict of interest.

\section{References}

1. Ramos-Levi AM, Marazuela M. Pathogenesis of thyroid autoimmune disease: the role of cellular mechanisms. Endocrinol Nutr 2016; 63 (8): 421-429.

2. Fromm GA, Lascano EF, Burr GE, et al. Tiroiditis cronica inespecifica. Rev Assoc Med Arg, 1953; 67: 162-170.

3. Roitt IM, Doniach D, Campbell PN, Hudson RV. Auto-antibodies in Hashimoto's disease (lymphadenoid goitre). Lancet 1956; 271 (6947): 820-821.

4. Ajjan RA, Watson PF, McIntosh RS, Weetman AP. Intrathyroidal cytokine gene expression in Hashimoto's thyroiditis. Clin Exp Immunol 1996; 105 (3): 523-528.

5. Nakagawa T, Tsuruoka M, Ogura H, et al. IL-6 positively regulates Foxp3+CD8+ T cells in vivo. Int Immunol 2010; 22 (2): 129-139.
6. Palazzo FF, Hammond LJ, Goode AW, Mirakian R. Death of the autoimmune thyrocyte: is it pushed or does it jump? Thyroid 2000; 10 (7): 561-572.

7. Rose-John S, Scheller J, Elson G, Jones SA. Interleukin-6 biology is coordinated by membrane-bound and soluble receptors: role in inflammation and cancer. J Leukoc Biol 2006; 80 (2): 227-236.

8. Weetman AP, Bright-Thomas R, Freeman M. Regulation of interleukin-6 release by human thyrocytes. J Endocrinol 1990; 127 (2): 357-361.

9. Ruggeri RM, Sciacchitano S, Vitale A, et al. Serum hepatocyte growth factor (HGF) is increased in Hashimoto's thyroiditis whether or not it is associated with nodular goiter as compared with healthy non-goitrous individuals. J Endocrinol Invest 2009; 32 (5): 465-469.

10. El-Shenawy SZ, Hemi MH, Attia H. Serum levels of proinflammatory cytokines (interleukin $6 \&$ interleukin 15) and adiponectin in Hashimoto's thyroiditis with different Thyroid function states. Am J Sci 2011; 7 (6): 1156-1162.

11. Chen RH, Chang CT, Chen WC, et al. Proinflammatory cytokine gene polymorphisms among Hashimoto's thyroiditis patients. J Clin Lab Anal 2006; 20 (6): 260-265.

12. Rekha PL, Ishaq M, Valluri V. A differential association of interferon-gamma high-producing allele $\mathrm{T}$ and low-producing allele A (+874 A/T) with Hashimoto's thyroiditis and Graves' disease. Scand J Immunol 2006; 64 (4): 438-443.

13. Inoue $\mathrm{N}$, Watanabe $\mathrm{M}, \mathrm{Nanba} \mathrm{T}$, et al. Involvement of functional polymorphisms in the TNFA gene in the pathogenesis of autoimmune thyroid diseases and production of anti-thyrotropin receptor antibody. Clin Exp Immunol 2009; 156 (2): 199-204.

14. Lacka K, Paradowska-Gorycka A, Maciejewski A, et al. Interleukin 1 Beta (ILlbeta) gene polymorphisms (SNP-511 and SNP+3953) in Hashimoto's thyroiditis among the Polish population. Exp Clin Endocrinol Diabetes 2014; 122 (9): 544-547.

15. Rasmussen AK, Bendtzen K, Feldt-Rasmussen U. Thyrocyte-interleukin-1 interactions. Exp Clin Endocrinol Diabetes 2000; 108 (2): 67-71.

16. Rasmussen AK. Cytokine actions on the thyroid gland. Dan Med Bull 2000; 47 (2): 94-114.

17. Lei Y, Yang J, Li H, et al. Changes in glucose-lipid metabolism, insulin resistance, and inflammatory factors in patients with autoimmune thyroid disease. J Clin Lab Anal 2019; 33 (7): e22929.

18. Aust G, Heuer M, Laue S, et al. Expression of tumour necrosis factor-alpha (TNF-alpha) mRNA and protein in pathological thyroid tissue and carcinoma cell lines. Clin Exp Immunol 1996; 105 (1): 148-154.

19. Caturegli P, De Remigis A, Rose NR. Hashimoto thyroiditis: clinical and diagnostic criteria. Autoimmun Rev 2014; 13 (4-5): 391-397.

\section{AUTHORS' CONTRIBUTIONS}

JS and KD prepared a first draft of the article. All authors participated in preparing the final version of the publication. 